\title{
Analysis of Kinematic Motion Deviations of Rotary Tables Based on Geometric Tolerances*
}

\author{
Nobuhiro SUGIMURA**, Hitomi WATABIKI***, Wiroj THASANA****, \\ Koji IWAMURA** and Yoshitaka TANIMIZU** \\ ** Osaka Prefecture University \\ 1-1 Gakuen-cho, Naka-ku, Sakai, Japan \\ E-mail: sugimura@me.osakafu-u.ac.jp \\ ${ }^{* * *}$ Yamazaki Mazak Co. \\ 1-131 Takeda, Oguchi-cho, Niwa-gun, Aichi, Japan \\ **** Thai-Nichi Institute of Technology \\ 1771/1 Pattanakarn Rd., Suanluang, Bangkok, Thailand
}

\begin{abstract}
Machine tools are recognized as key elements of manufacturing systems, and product quality and cost mainly depend on performances of the machine tools. Much progress has been made in the machine tool technologies, aimed at improving the performances of the machine tools from various viewpoints, such as accuracy, reliability, productivity, and flexibility. The machining accuracy is one of the most important characteristics of the machine tools. From the viewpoints of the design and the manufacturing of the machine tools and their components, one of the important issues is to clarify the relationships between the kinematic motion deviations of the machine tools and the geometric tolerances of the components, such as the guide ways and the bearings. The objectives of the present research are to establish mathematical models representing the kinematic motion deviations of the machine tools, on the basis of the geometric tolerances of the components, and to apply the models to theoretical analysis of the kinematic motion deviations of the machine tool components.
\end{abstract}

Key words: Machine Tools, Machining Errors, Kinematic Motion Deviation, Geometric Tolerances, Rotary Tables

\section{Introduction}

One of the most important revolutions in the later part of the last century is introduction of NC (Numerical Control) machine tools, which are able to carry out various complicated machining processes without human interaction. Various types of NC machine tools are now being designed and applied to machining processes of complicated machine products. The machining accuracy is one of the most important characteristics of the NC machine tools for generating the products with the high accuracy and the complicated geometries.

Some researches have been carried out to analyze the machining accuracy of the machine tools based on the deviations of the shape generation motions between the tools and the workpieces ${ }^{(1-4)}$. However, the kinematic motion deviations of the machine tools are deeply influenced by the geometric deviations of the components, such as guide ways and bearings. Therefore, it is now required to clarify the relationships between the kinematic motion deviations of the machine tools and the geometric deviations of the components, from the viewpoints of the design and the manufacturing of the machine tools and their

${ }^{*}$ Received 11 June, 2012 (№. 12-0256) [DOI: 10.1299/jamdsm.6.1132]

Copyright $(2012$ by JSME 
components.

As regards the geometric deviations of the machine components, many researches have been carried out to deal with the dimensional tolerances and the geometric tolerances, aimed at realizing systematic analysis and design methodologies for the three dimensional machine products ${ }^{(5-8)}$. However, the relationships between the kinematic motion deviations and the geometric deviations of the components have not yet been clarified, due to that the linear and rotary tables of the machine tools are supported by more than tow guide ways and that all the geometric deviations of individual guide ways affect the kinematic motion deviations of the linear and rotary tables.

The objectives of the present research are to establish mathematical models representing the kinematic motion deviations of the machine tools, on the basis of the geometric tolerances of the components, and to apply the models to theoretical analysis of the kinematic motion deviations of the machine tools. In the previous papers ${ }^{(9,10)}$, a mathematical model has been proposed to represent the kinematic motion deviations of the linear tables and the proposed model was applied to the analysis of the kinematic motion deviations of the linear tables and the 3-axis machining centers. However, the rotary tables have not yet been discussed in detail ${ }^{(11)}$. The kinematic motion deviations of the rotary tables are not linear against the rotational angles of the tables. That is, the kinematic motion deviations are represented by the formulas including the trigonometric functions.

Main issues discussed in the present paper are summarized as follows.

(1) Modeling of geometric deviations of guide ways.

(2) Modeling of geometric deviations of rotary tables.

(3) Analysis of kinematic motion deviations of rotary tables.

\section{Geometric Deviations of Components and Kinematic Motion Deviations of Machine Tools}

The shape generation processes of the machine tools are carried out by the shape generation motions between the tools and the workpieces, and the shape generation motions are realized by a set of the relative motions between the constituting components. Therefore, the kinematic motion deviations of the shape generation motions are influenced by the geometric deviations of the components and the kinematic motion deviations between these components.

Figure 1 summarizes the relationships between the kinematic motion deviations of the shape generation motions and the deviations of the components. In the figure, the nodes WO, CT and Fij represent the workpieces to be machined, the tools, and the geometric features connecting the rigid components Unit-i of the machine tools, respectively. The features mean here the geometric elements, such as plane faces of guide ways and cylindrical faces of bearings, which connect and constrain the relative motions between the pairs of the units.

The kinematic motion deviations of the shape generation motions of the machine tools are defined as the kinematic motion deviations between the tools CT against the workpieces WO, and the deviations are influenced by various types of deviations, such as the position deviations in setting up the tools and the workpieces, the relative position deviations between the units, and the geometric deviations of the features connecting the constituent units.

The objective of the present research is to represent and to analyze the kinematic motion deviations of the tools against the workpieces, based on the geometric deviations of the components. In particular, emphasis is given to modeling and analysis of the effects of the geometric deviation of the features on the kinematic motion deviations of the tools against the workpieces. 


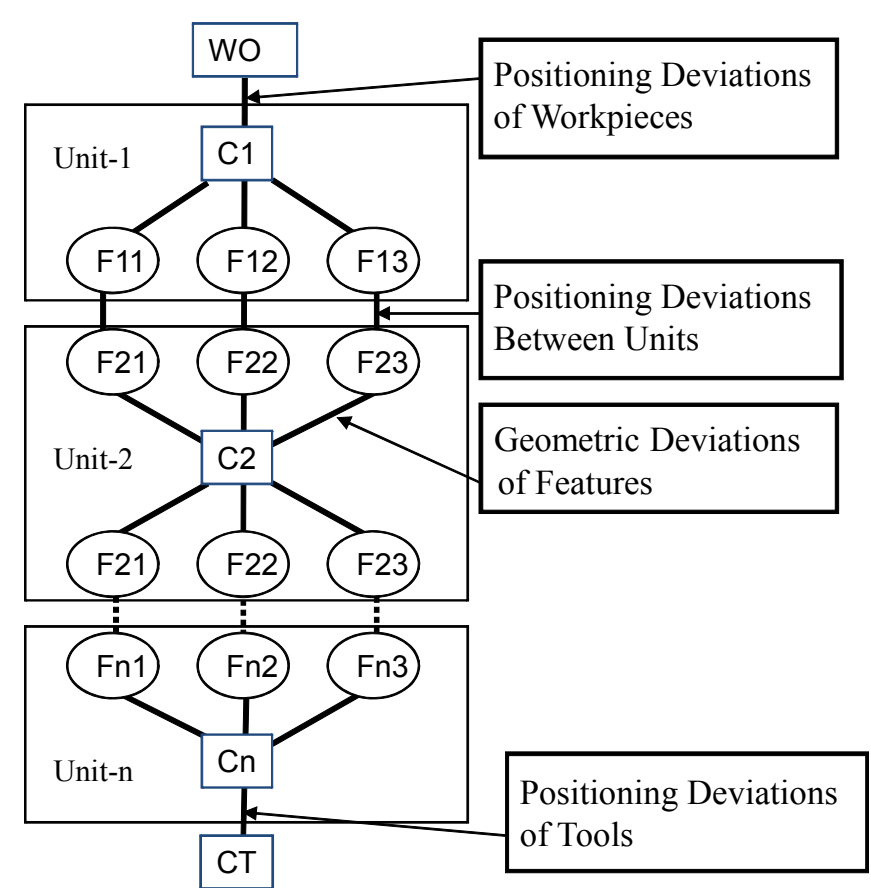

Fig. 1 Relationships between kinematic motion deviations and deviations of components.

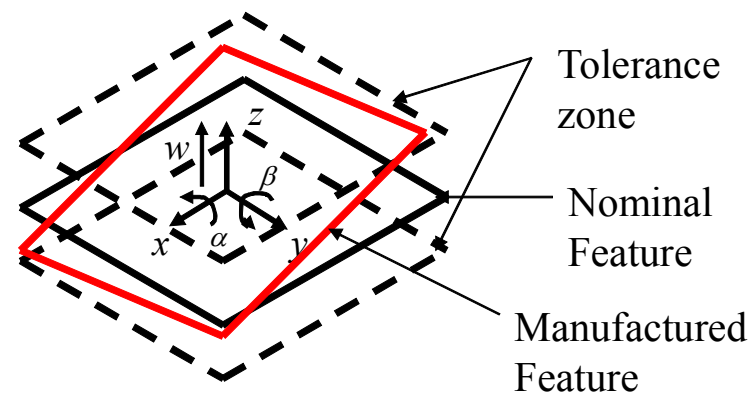

Fig. 2 Definition of geometric tolerance of plane features.

\section{Geometric Deviations of Features ${ }^{(12-13)}$}

The geometric tolerances of the features specify the allowable areas named "tolerance zones," which constrain the position and orientation deviations of the associated features against the nominal features, as shown in Fig. 2. The associated features and the nominal features mean the features of the manufactured products and the ideal features defined in the design phase, respectively. The geometric deviations of the associated features from the nominal features are represented by sets of parameters named "deviation parameters ${ }^{(11)}$." For example, one position parameter $w$ and two rotational parameters $\alpha$ and $\beta$ are required to represent the geometric deviations of the associated plane features against the nominal plane features, for the case where the tolerance zone is given by the area between a pair of parallel planes.

In the research, the followings are assumed for the ease of the modeling and the analysis of the geometric deviations.

(1) The deviation parameters $\delta_{i}$ representing the position and orientation deviations of the associated features follow the normal distribution $N\left(\mu_{i}, \sigma_{i}\right)$, and $\mu_{i}=0$. Where, $\mu_{i}$ and $\sigma_{i}$ are the mean values and the standard deviations, respectively. 
(2) The manufacturing processes of the components are well controlled, and the proportion of the non-conforming components is as small as a value $P d$ called "percent defective". Here, the non-conforming components mean the components, in which the toleranced features exceed the tolerance zones.

(3) Equation (1) represents the relationships between the standard deviations $\sigma_{i}$ of the deviation parameters of the toleranced features and the sizes of the tolerance zones.

$$
\sigma_{i}=\delta_{\text {imax }} / C_{P d}
$$

where,

$\delta_{\text {imax }}$ : maximum values of the deviation parameters $\sigma_{i}$ if the other deviation parameters $\delta_{j}=$ $0,(i \neq j)$.

$C_{P d}$ : a constant representing the ratio of the maximum values $\delta_{\text {imax }}$ and the standard deviations $\sigma_{i}$.

Let us consider a case shown in Fig. 2, as an example. The maximum values $\delta_{\text {imax }}$ are given as follows.

$$
\begin{aligned}
& \delta_{1}=w, \delta_{2}=\alpha, \delta_{3}=\beta \\
& \delta_{1 \max }=t / 2, \delta_{2 \max }=t / L_{1}, \delta_{3 \max }=t / L_{2}
\end{aligned}
$$

where,

$L_{1}, L_{2}$ : Length and width of the plane feature.

$t$ :Tolerance values, e.g. the distance between two planes representing the tolerance zones.

The following equation gives the conditions that the plane features are included within the tolerance zone between a pair of planes.

$$
-t / 2<\delta_{1}+L_{1} \delta_{2} / 2+L_{3} \delta_{3} / 2<t / 2
$$

The probability that the toleranced features are included within the tolerance zones is given by the following equation.

$$
1-P d=\left(\frac{2}{\sqrt{2 \pi}}\right)^{3} \int_{0}^{C_{P d}} \int_{0}^{C_{P d}-x_{1}} \int_{0}^{C_{P d}-x_{1}-x_{2}}\left(\prod_{i=1}^{3} \exp \left(-\frac{x_{i}^{2}}{2}\right)\right) \mathrm{d} x_{3} \mathrm{~d} x_{2} \mathrm{~d} x_{1}
$$

where,

$$
x_{1}=2 C_{P_{d}} \delta_{1} / t, x_{2}=L_{1} C_{P_{d}} \delta_{2} / t, x_{3}=L_{2} C_{P_{d}} \delta_{3} / t
$$

If the percent defective $P d$ is set to be $0.27 \%$, the constant $C_{P d}$ can be estimated as " $C_{P d}=5.83$," through the numerical analysis of Eq. (4). The standard deviations $\sigma_{i}$ of deviation parameters $\delta_{i}$ are also obtained based on the $C_{P d}$, the dimensions of the plane features and the size of tolerance zone $t$, by applying Eqs. (1) and (2).

In the case of an axis feature shown in Fig. 3, the following equation gives the deviation parameter and their maximum values.

$$
\begin{aligned}
& \delta_{1}=u, \delta_{2}=v, \delta_{3}=\alpha, \delta_{4}=\beta \\
& \delta_{1 \max }=\delta_{2 \max } t / 2, \delta_{3 \max }=\delta_{4 \max }=t / \ell
\end{aligned}
$$

The following conditins are required to safisfy the geometric tolerance.

$$
\left(\frac{\ell}{2} \beta+u\right)^{2}+\left(\frac{\ell}{2} \alpha+v\right)^{2} \leq\left(\frac{t}{2}\right)^{2},\left(\frac{\ell}{2} \beta-u\right)^{2}+\left(\frac{\ell}{2} \alpha-v\right)^{2} \leq\left(\frac{t}{2}\right)^{2}
$$

In this case, the $C_{P d}$ is estimated as " $C_{P d}=5.06$," if the percent defective $P d$ is set to be 


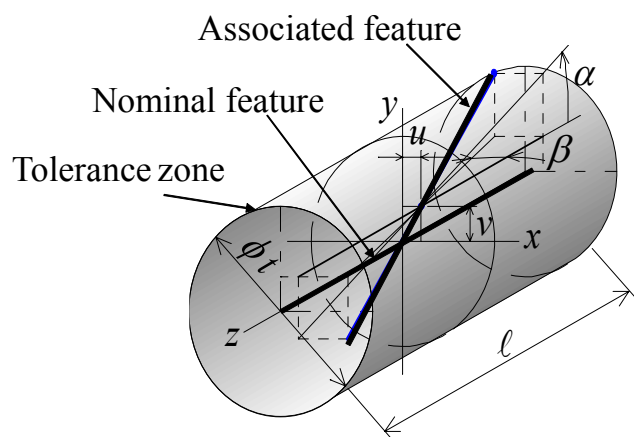

Fig. 3 Definition of geometric tolerance of axis features.

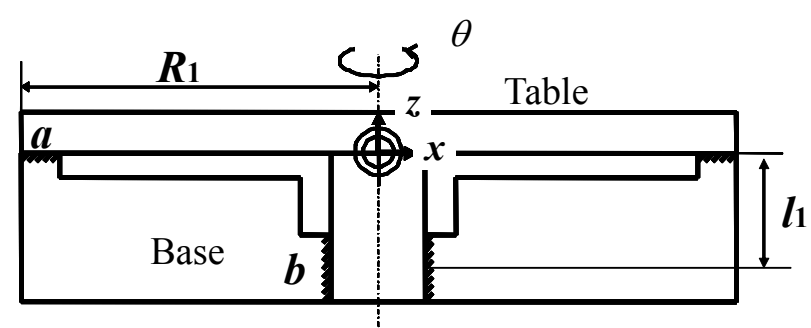

(a) Rotary table with vertical axis.

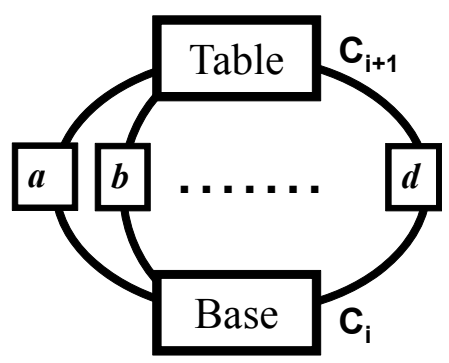

(b) Guide ways between table and base.

Fig. 4 Rotary table and its connecting relationships.

$0.27 \%$. The $C_{P d}$ value obtained here is same in the case that a cylindrical feature is constrained by a pair of co-axial cylinders. The $C_{P d}$ values for plane features and axis features obtained here will be applied for the following sections.

\section{Geometric Deviations of Rotary Tables}

Figure 4 (a) shows a typical example of rotary tables with a vertical axis utilized for the machine tools. A base supports and guides a rotary table by a plane face and a cylindrical face called guide ways and bearings, which are indicated by $\boldsymbol{a}$ and $\boldsymbol{b}$. In the case, the relative position and orientation of the table against the base are given by the following equation, if the guide ways coincide with the nominal features.

$$
x_{i}=A_{a} A_{a},{ }^{-1} x_{i+1}=A_{b} A_{b},{ }^{-1} x_{i+1}
$$

where,

$\mathbf{x}_{\mathrm{i}}$ : Position vector of a point $\mathbf{P}$ in the base coordinate system $\mathbf{C}_{\mathrm{i}}$.

$\mathbf{x}_{i+1}$ : Position vector of $\mathbf{P}$ in the table coordinate system $\mathbf{C}_{i+1}$.

$\mathbf{A}_{\mathrm{j}}(\mathrm{j}=\boldsymbol{a}, \boldsymbol{b}): 4 \times 4$ homogeneous transformation matrices representing the positions and orientations of the guide ways $\mathrm{j}$ in the base coordinate system $\mathbf{C}_{\mathrm{i}}$.

$\mathbf{A}_{\mathrm{j}}{ }^{\prime}(\mathrm{j}=\boldsymbol{a}, \boldsymbol{b}): 4 \times 4$ homogeneous transformation matrices representing the positions and orientations of the guide ways $\mathrm{j}$ in the table coordinate system $\mathbf{C}_{i+1}$. 


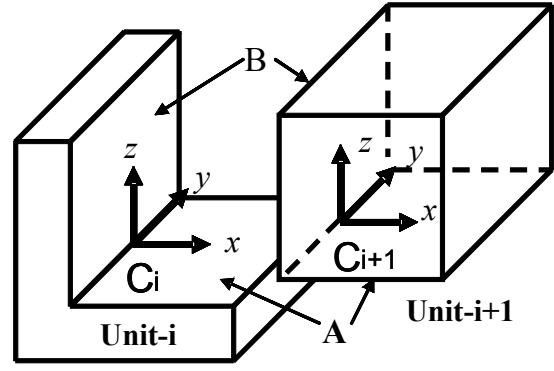

Priority A $>\mathbf{B}$

(a) Priority between connection features

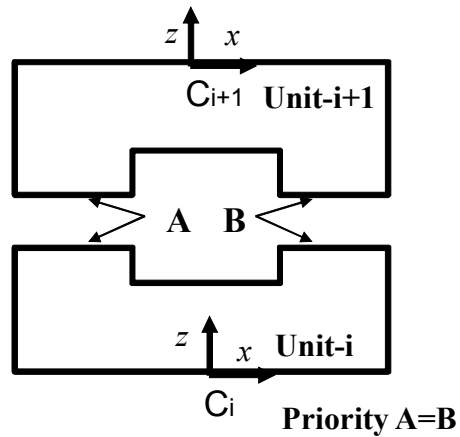

(b) No priority between connecting features

Fig. 5 Features connecting a pair of units

However, the relative positions of the table against the base are influenced by the position and orientation deviations of the guide ways, if the guide ways do not coincide with the nominal features. The positions of the table against the base are represented by the following equation corresponding to the individual guide ways.

$$
\mathbf{X}_{\mathrm{i}}=\mathbf{A}_{\mathrm{j}} \mathbf{D}_{\mathrm{j}} \mathbf{D}_{\mathrm{j}}{ }^{,-1} \mathbf{A}_{\mathrm{j}}{ }^{-1} \mathbf{X}_{\mathrm{i}+1} \quad(\mathrm{j}=\boldsymbol{a}, \boldsymbol{b})
$$

where,

$\mathbf{D}_{\mathrm{j}}, \mathbf{D}_{\mathrm{j}}$ : $4 \mathrm{x} 4$ homogeneous transformation matrices representing the position and orientation deviations of the pair of features constituting the guide ways $j$.

Equation (8) means that various values of the relative position of the table against the base are obtained for the individual guide ways, as shown in Fig. 4(b). A systematic method is therefore required to evaluate one value of the relative position for the cases where the guide ways have the geometric deviations. It is because that both the base and the table are the rigid bodies and the relative position between the rigid bodies should be represented by one value. The following two methods are proposed here to solve the problem.

(1) Priority among connections

Let us consider the case shown in Fig. 5 (a) as an example. The Unit-i and the Unit-i+1 is connected by two set of the features indicated by $\mathbf{A}$ and $\mathbf{B}$, which are perpendicular to each other. The deviations of the relative positions of the Unit-i+1 to the Unit-i, which are specified by $\mathbf{D}_{\mathbf{j}} \mathbf{D}_{\mathbf{j}}{ }^{-1}$ in Eq. (8), are given by the following equations for the individual sets of the connecting features.

$$
\begin{aligned}
\mathbf{D}_{A} \mathbf{D}_{A^{\prime}}{ }^{-1} & =\left(\begin{array}{cccc}
1 & 0 & \delta \beta A & 0 \\
0 & 1 & -\delta \alpha A & 0 \\
-\delta \beta A & \delta \alpha A & 1 & \delta w A \\
0 & 0 & 0 & 1
\end{array}\right) \\
\mathbf{D}_{B} \mathbf{D}_{B^{\prime}}{ }^{-1} & =\left(\begin{array}{cccc}
1 & -\delta \gamma B & 0 & \delta w B \\
\delta \gamma B & 1 & -\delta \alpha B & 0 \\
0 & \delta \alpha B & 1 & 0 \\
0 & 0 & 0 & 1
\end{array}\right)
\end{aligned}
$$

In this case, two values are obtained for defining the relative positions of the Unit-i and the Unit-i+1. Priority may be set to the connecting features, based on the areas of the features and the direction of the gravity. Higher priority given to the feature set $\mathbf{A}$ in the case, if the direction of the gravity is $-\mathrm{z}$. Therefore, the deviations of the relative positions of the Unit- $i+1$ to the Unit-i are given as follows. 
$\mathbf{D}_{A B} \mathbf{D}_{A B^{\prime}}{ }^{-1}=\left(\begin{array}{cccc}1 & -\delta \gamma B & \delta \beta A & \delta w B \\ \delta \gamma B & 1 & -\delta \alpha A & 0 \\ -\delta \beta A & \delta \alpha A & 1 & \delta w A \\ 0 & 0 & 0 & 1\end{array}\right)$

(if $\mathbf{A}>\mathbf{B}$ )

where, "A > B" specifies that the feature set $\mathbf{A}$ has higher priority to the feature set $\mathbf{B}$.

(2) Non-priority among connections

For the case shown in Fig. 5 (b), there are two sets of the connecting features between the Unit-i and the Unit-i+1. However, there is not any priority between the feature sets $\mathbf{A}$ and $\mathbf{B}$. In this case, the deviations for the both connecting feature sets are averaged based on the least square method ${ }^{(10)}$, and the deviations of the relative position of the Unit-i+1 to the Unit-i are given as follows.

$$
\mathbf{D}_{A B} \mathbf{D}_{A B}{ }^{-1}=\left(\begin{array}{cccc}
1 & 0 & (\delta \beta A+\delta \beta B) / 2 & 0 \\
0 & 1 & -(\delta \alpha A+\delta \alpha B) / 2 & 0 \\
-(\delta \beta A+\delta \beta B) / 2 & (\delta \alpha A+\delta \alpha B) / 2 & 1 & \left(\delta_{v B}+\delta_{v B}\right) / 2 \\
0 & 0 & 0 & 1
\end{array}\right)
$$

(if $\mathbf{A}=\mathbf{B}$ )

where, "A = B" means that the feature sets $\mathbf{A}$ and $\mathbf{B}$ do not have any priority.

Figure 6 shows a typical example of rotary tables with a horizontal axis utilized for the machine tools. In this case, same method is applicable.

\section{Analysis of Kinematic Motion Deviations of Rotary Tables}

\subsection{Modeling of kimematic motion deviations}

The geometric deviations of the rotary tables shown in Fig. 4 (a), are formulated based on the geometric deviations of the guide ways, by applying the method described in Section 4. Equation (12) is obtained to describe the kinematic motion deviations of the rotary table, on the assumption that the priority among the guide ways is " $\boldsymbol{a}>\boldsymbol{b}$."

$$
A_{6}(\theta)=\left(\begin{array}{cccc}
\cos \theta & -\sin \theta & \delta_{V \beta 1} & \delta_{V x} \\
\sin \theta & \cos \theta & \delta_{V \alpha 1} & \delta_{V y} \\
\delta_{V \beta 2} & \delta_{V \alpha 2} & 1 & \delta_{V z} \\
0 & 0 & 0 & 1
\end{array}\right)
$$

where,

$\theta$ : Rotational angle of the table.

$\delta_{V \alpha 1}=-\beta_{b 2} \sin \theta+\alpha_{b 2} \cos \theta-\alpha_{b 1}$

$\delta_{V \alpha 2}=\beta_{b 1} \sin \theta+\alpha_{b 1} \cos \theta-\alpha_{b 2}$

$\delta_{V \beta 1}=-\beta_{b 2} \cos \theta-\alpha_{b 2} \sin \theta+\beta_{b 1}$

$\delta_{V \beta 2}=-\beta_{b 1} \cos \theta+\alpha_{b 1} \sin \theta+\beta_{b 2}$

$\delta_{V x}=-l_{1} \beta_{b 2} \cos \theta-l_{1} \alpha_{b 2} \sin \theta+l_{1} \beta_{b 1}-\delta x_{b 2} \cos \theta+\delta y_{b 2} \sin \theta+\delta x_{b 1}$

$\delta_{V y}=-l_{1} \beta_{b 2} \sin \theta+l_{1} \alpha_{b 2} \cos \theta-l_{1} \alpha_{b 1}-\delta x_{b 2} \sin \theta-\delta y_{b 2} \cos \theta+\delta y_{b 1}$

$\delta_{V z}=\delta z_{a 1}-\delta z_{a 2}$ 


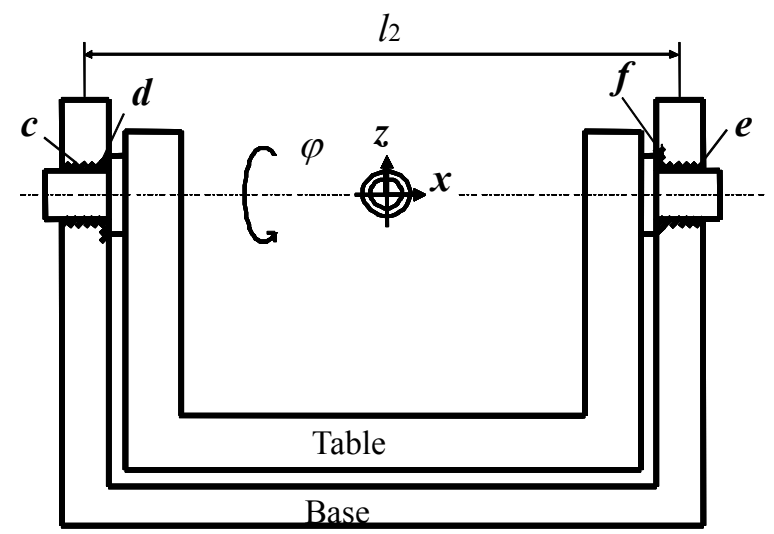

Fig. 6 Rotary table with horizontal axis

The kinematic motion deviations of the rotary tables with horizontal axis shown in Fig. 6 are also formulated as shown in the following equation.

$$
A_{4}(\varphi)=\left(\begin{array}{cccc}
1 & \frac{1}{2} \delta_{H \gamma 1} & \frac{1}{2} \delta_{H \beta 1} & \frac{1}{2} \delta_{H x} \\
\frac{1}{2} \delta_{H \gamma 2} & \cos \varphi & -\sin \varphi & \frac{1}{2} \delta_{H y} \\
\frac{1}{2} \delta_{H \beta 2} & \sin \varphi & \cos \varphi & \frac{1}{2} \delta_{H z} \\
0 & 0 & 0 & 1
\end{array}\right)
$$

where,

$\varphi$ : Rotational angle of the table.

$\delta_{H \beta 1}=-\beta_{c 2}-\beta_{e 2}+\left(\gamma_{c 1}+\gamma_{e 1}\right) \sin \varphi+\left(\beta_{c 1}+\beta_{e 1}\right) \cos \varphi$

$\delta_{H \beta 2}=-\beta_{c 1}-\beta_{e 1}-\left(\gamma_{c 2}+\gamma_{e 2}\right) \sin \varphi+\left(\beta_{c 2}+\beta_{e 2}\right) \cos \varphi$

$\delta_{H \gamma 1}=\gamma_{c 2}+\gamma_{e 2}-\left(\gamma_{c 1}+\gamma_{e 1}\right) \cos \varphi+\left(\beta_{c 1}+\beta_{e 1}\right) \sin \varphi$

$\delta_{H \gamma 2}=\gamma_{c 1}+\gamma_{e 1}-\left(\gamma_{c 2}+\gamma_{e 2}\right) \cos \varphi-\left(\beta_{c 2}+\beta_{e 2}\right) \sin \varphi$

$\delta_{H x}=\delta x_{d 1}-\delta x_{d 2}+\delta x_{f 1}-\delta x_{f 2}$

$\delta_{H y}=\delta y_{c 1}+\delta y_{e 1}-\left(\delta y_{c 2}+\delta y_{e 2}\right) \cos \varphi+\left(\delta z_{c 2}+\delta z_{e 2}\right) \sin \varphi$

$\left.-\frac{l_{2}}{2}\left\{\left(\gamma_{e 1}-\gamma_{c 1}\right)-\left(\gamma_{e 2}-\gamma_{c 2}\right) \cos \varphi-\left(\beta_{e 2}-\beta_{c 2}\right) \sin \varphi\right)\right\}$

$\delta_{H z}=\delta z_{c 1}+\delta z_{e 1}-\left(\delta z_{c 2}+\delta z_{e 2}\right) \cos \varphi-\left(\delta y_{c 2}+\delta y_{e 2}\right) \sin \varphi$

$\left.+\frac{l_{2}}{2}\left\{\left(\beta_{e 1}-\beta_{c 1}\right)-\left(\beta_{e 2}-\beta_{c 2}\right) \cos \varphi+\left(\gamma_{e 2}-\gamma_{c 2}\right) \sin \varphi\right)\right\}$

\subsection{Analysis of kinematic motion deviations of two-axis rotary tables}

A two-axis rotary table shown in Fig. 7 is designed by combining two tables shown in Figs. 4(a) and 6, and the kinematic motion deviations of the table are estimated based on the formulas presented in Eqs. (12) and (13). The important conditions of the tables are assumed as follows:

(1) Rotary table with vertical axis

- Table sizes in Fig. 4 (a):

$l_{1}=20[\mathrm{~mm}], l_{2}=70[\mathrm{~mm}], l_{3}=30[\mathrm{~mm}], R_{1}=250[\mathrm{~mm}]$ 


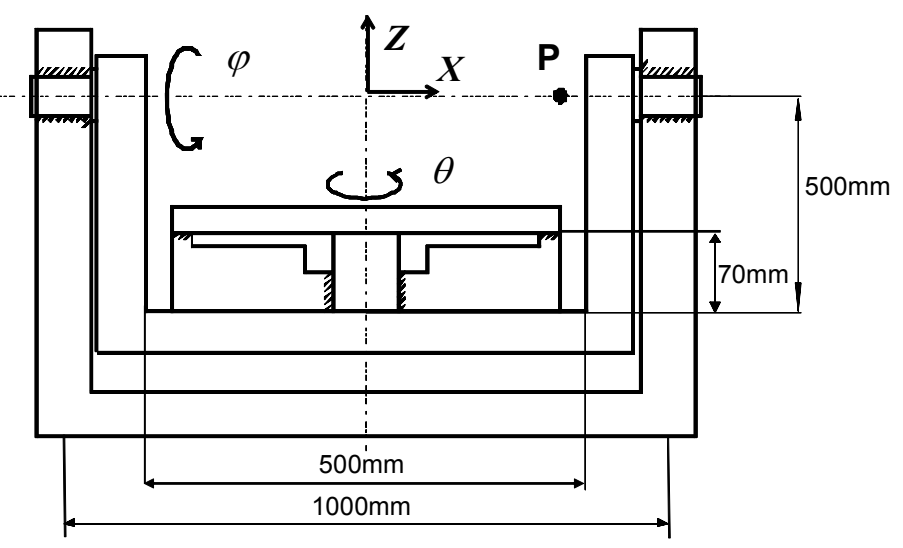

Fig. 7 Two-axis rotary table

- Standard deviations of all the deviation parameters $\delta$ in Eq. (12).

Position deviations $=1 \times 10^{-3}[\mathrm{~mm}]$

Orientation deviations $=1 \times 10^{-3}[\mathrm{rad}$.]

(2) Rotary table with horizontal axis

- Table sizes in Fig. 6:

$$
l_{4}=500[\mathrm{~mm}], R_{2}=1,000[\mathrm{~mm}]
$$

- Standard deviations of all the deviation parameters $\delta$ in Eq. (13).

Position deviations $=1 \times 10^{-3}[\mathrm{~mm}]$

Orientation deviations $=1 \times 10^{-3}[\mathrm{rad}$. $]$

The kinematic motion deviation parameters in Eqs. (12) and (13) are given by both the mean values and the standard deviations, therefore, all the deviation parameters in the transformation matrices are estimated by applying the following equations.

$$
\begin{aligned}
& \mathbf{C}=\mathbf{A B}, \quad \mathbf{C}=\left\{c_{i j}\right\}, \mathbf{A}=\left\{a_{i j}\right\}, \mathbf{B}=\left\{b_{i j}\right\} \\
& \mu_{i j}^{c}=\sum_{k=1}^{4} \mu_{i k}^{a} \mu_{k j}^{b} \\
& \sigma_{i j}^{c}=\sqrt{\sum_{k=1}^{4}\left\{\left(\mu_{i k}^{a} \sigma_{k j}^{b}\right)^{2}+\left(\mu_{k j}^{b} \sigma_{i k}^{a}\right)^{2}\right\}}
\end{aligned}
$$

where,

$\mu_{i j}^{c}, \mu_{i k}^{a}, \mu_{k j}^{b}$ : Mean values of the elements of transformation matrices A, B and C.

$\sigma_{i j}^{c}, \sigma_{i k}^{a}, \sigma_{k j}^{b}$ : Standard deviations of the elements of transformation matrices A, B and C.

Kinematic motion deviations of a point $P$ in Fig. 7 is estimated by applying Eqs. (12), (13) and (14). The point $P$ is selected as a point that clearly presents the effects of the kinematic motion deviations in the rotational motion around the $\mathrm{X}$ and $\mathrm{Z}$ axes. The initial position of $P$ is set that $P$ coincides with the rotational axis of $X$ axis and that the rotational radius around the $\mathrm{Z}$ axis is largest. The kinematic deviations in $\mathrm{X}-\mathrm{Y}$ plane and ones in $\mathrm{Z}-\mathrm{X}$ plane are presented in Figs. 8 (a) and (b), respectively. In the figure, the crossing points of a pair of line segments give the mean values of the kinematic motion of the point $P$, and the vertical and horizontal line segments show the standard deviations in $\mathrm{X}$-, Y- and 


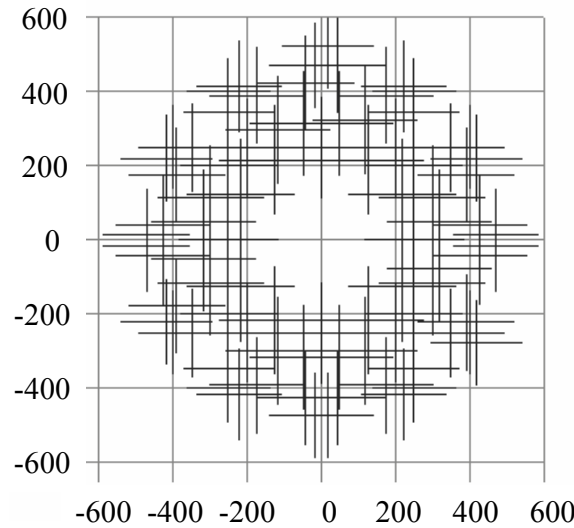

(a) X-Y plane

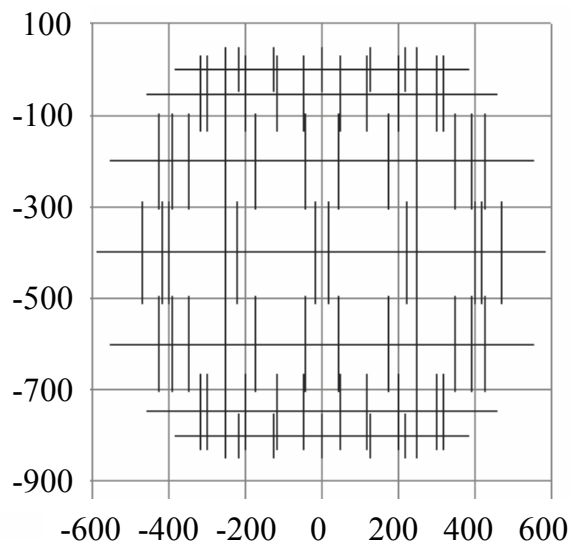

(b) Z-X plane

Fig. 8 Mean values and standard deviations of kinematic motion of point $P$

(Standard deviations are magnified by 100)

Z-directions of the kinematic motion of the point $P$. The standard deviations are magnified by 100 for the ease of understand. It is understood that the kinematic motion deviations are evaluated based on the mean values and the standard deviations in X-, Y- and Z-directions.

The kinematic motion deviations of any points on the table are evaluated by applying the proposed methods. In the future works, the model will be expanded to represent the five-axis machine tools, aimed at analyzing the kinematic motion deviations of the cutting points on the tools against the workpieces.

\section{Conclusions}

A mathematical model has been proposed to represent the kinematic motion deviations of the rotary tables, and the proposed model was applied to the analysis of the kinematic motion deviations of the two-axis rotary table required to analyze the 5-axis machining centers. Following remarks are concluded.

(1) A systematic method is proposed to establish mathematical model representing the kinematic motion deviations of the machine tools, based on the geometric tolerances of the components.

(2) The geometric deviations of the rotary tables are discussed, and a method is proposed to estimate the geometric deviations of the rotary tables, considering the priorities among the guide ways and the bearings of the rotary tables. A two-axis rotary table model is established to represent and to analyze the kinematic motion deviations of the complicated machine tools like five-axis machining centers. The proposed models will be combined with the model of the linear tables presented in the previous papers ${ }^{(9)-(11)}$.

(3) The kinematic motion deviations of the two-axis rotary tables are estimated by applying the proposed model. The proposed method provides us with a systematic method to analyze and to evaluate the kinematic motion deviations of the rotary tables based on both the mean values and the standard deviations of the points.

\section{References}

(1) Sugimura, N., Iwata, K., and Oba, F., Formation of shape generation processes of machine tools, Proc. of IFAC' 81, Vol. XIV (1981), pp.158-163.

(2) Reshetov, D. N., and Portman, V. T., 1988, Accuracy of machine tools, ASME Press (1998).

(3) Sakamoto, S. and Inasaki, I., Error analysis of precision machine tools, Proc. of the 3rd Int. 
Conf. of Ultraprecision in Manufacturing Engineering (1994), pp.245-250.

(4) Sugimura, N. and Mitani, S., Analytical approach for design of alignment errors of machine tools, Proc. of 1998 Pacific Conference on Manufacturing (1998), pp.371-374.

(5) Roy, R. Liu, C.R. and Woo, T.C., Review of dimensioning and tolerancing: representation and processing, Computer-Aided Design, 23 (1991), pp.466-483.

(6) Voelcker, H.B., A current perspective on tolerancing and metrology, CRTD-Vol.27, Int. Forum on Dimensional Tolerancing and Metrology (1993), pp.46-68.

(7) Nigam, S.D., and Turner, J. U., Review of statistical approaches to tolerance analysis, Computer-Aided Design, 27, 1 (1995), pp.6-15.

(8) Ngoi, B.K.A., Lim, B.H. and Ang, P.S., Nexus method for stack analysis of geometric dimensioning and tolerancing (GDT) problems, Int. J. Prod. Res., 38, 1 (2000), pp.21-37.

(9) Satonaka, N., Sugimura, N., Tanimizu, Y., amd Iwamura, K., Analysis of kinematic motion deviations of machining centers based on geometric tolerances, Proc. of the 4th LEM-21 (2007), pp.629-634.

(10) Satonaka, N., Sugimura, N., Tanimizu, Y., and Iwamura, K., A study on modeling and analysis of kinematic motion deviations of machine tools (2nd Report, Modeling and analysis of linear tables and machine tools, Trans. JSME, 74, 737 (2008), pp.198-205. (in Japanese)

(11) Watabiki, H., Sugimura, N., Tanimizu, Y. and Iwamura, K., Analysis of kinematic motion deviations of machining centers based on geometric tolerances, Proc. of 5th International Conference on Leading Edge Manufacturing in 21st Century(2009), pp.677-682.

(12) Sugimura, N., Nakamoto, K. and Satonaka, N., A study on statistical analysis of geometric tolerances of products, Proc. of the 17th International conference on Production Research (2003), CD-ROM, pp.1-6.

(13) Satonaka, N., Sugimura, N., Tanimizu, Y. and Iwamura, K., Mathematical analysis of three-dimensional geometric tolerance chains of mechanical products, Proc. of the 18th International Conference on Production Research (2005), CD-ROM, pp.1-6. 